\title{
COMPOSIÇÃO QUÍMICA DE GENÓTIPOS DE CANA-DE-AÇÚCAR EM DUAS IDADES, PARA FINS DE NUTRIÇÃO ANIMAL ${ }^{(1)}$
}

\author{
JOÃO BATISTA DE ANDRADE ${ }^{(2)}$; EVALDO FERRARI JUNIOR ${ }^{(2)}$; ROSANA APARECIDA POSSENTI ${ }^{(2)}$; \\ IVANI POZAR OTSUK ${ }^{(2)}$, LÉO ZIMBACK ${ }^{(3)}$; MARCOS GUIMARÃES DE ANDRADE LANDELL ${ }^{(4)}$
}

\begin{abstract}
RESUMO
Foi desenvolvido, em 1999/2000, no Instituto de Zootecnia, em Nova Odessa (SP), um experimento para avaliar 60 genótipos de cana-de-açúcar (24 cultivares e 36 clones), para fins de alimentação animal. O experimento foi executado em delineamento de blocos ao acaso, com três repetições. Os genótipos foram amostrados aos 12 e 18 meses de idade. Para todas as características analisadas: porcentagem de matéria seca (\%MS); de proteína bruta (\%PB); de extrato etéreo (\%EE); de matéria mineral (\%MM); de carboidratos totais não estruturais (\%CTN); de fibra insolúvel em detergente neutro (\%FDN); de fibra insolúvel em detergente ácido (\%FDA); de celulose (\%CEL); de hemicelulose (\%HEM); de lignina (\%LIG), verificaram-se diferenças entre os genótipos e as idades de corte, com exceção para \%EE, que não mostrou diferença entre as idades de corte. Para (\%CTN, \%NDF, \%ADF, \%CEL, \%HEM e \%LIG) foram observadas diferenças dos genótipos nas idades de corte.
\end{abstract}

Palavras-chave: carboidrato, fibra, proteína.

\section{ABSTRACT \\ CHEMICAL COMPOSITION OF SUGARCANE IN TWO AGES, FOR ANIMAL NUTRITION}

At the Instituto de Zootecnia, Nova Odessa (SP), Brazil, it was carried out an experiment to evaluate 24 cultivars and 36 sugarcane clones as raw material for animal feeding. The experimental design was set in randomized blocks with three replications. The sugarcanes were harvested at 12 and 18 months. Genotypes and age of harvest showed differences for \%DM (dry matter); \%CP (crude protein; \%EE (ether extract); \%ACH (ach); \% TNC (total non-structural carbohydrates) , \%NDF (neutral detergent fiber); \%ADF (acid detergent fiber); \%CEL (cellulose); \%HCEL (hemicellulose) and \%LIG (lignin), except for \%EE, for harvest ages. Significant differences for \%TNC, \%NDF, \%ADF, \%CEL, \%HCEL and \%LIG) were observed for two ages of harvest.

Key words: carbohydrate, fiber, protein.

$\left.{ }^{1}\right)$ Parte do projeto: Caracterização de cultivares de cana-de-açúcar, com vistas à alimentação animal. Financiado pela FAPESP. Recebido para publicação em 21 de novembro de 2002 e aceito em 24 de junho de 2004.

$\left(^{2}\right)$ Centro de Pesquisa e Desenvolvimento em Nutrição Animal e Pastagens, Instituto de Zootecnia, Caixa Postal 60, 13460-000 Nova Odessa (SP). E-mail: jbandrade@iz.sp.gov.br, ferrari@iz.sp.gov.br; possenti@iz.sp.gov.br; ivani@iz.sp.gov.br

$\left({ }^{3}\right)$ Núcleo de Pesquisa e Desenvolvimento do Jardim Botânico/Quarentenário, Instituto Agronômico de Campinas, Caixa Postal 28, 13001970 Campinas (SP). E-mail: lzimback@terra.com.br

(4) Centro Avançado de Pesquisa Tecnológica do Agronegócio de Cana, Instituto Agronômico, Caixa Postal 206, 14001-970 Ribeirão Preto (SP). E-mail: marcolandell@highnet.com 


\section{INTRODUÇÃO}

A cana-de-açúcar tem despertado o interesse dos pecuaristas, mais pelo seu alto potencial de produção do que pelo seu valor nutritivo, quando comparado, principalmente, com silagens de milho ou de sorgo. Nunes Junior (1987) ressalta que no Estado de São Paulo são freqüentes as usinas obterem rendimentos de 85 t.ha $^{-1}$ de colmo, o que corresponderia a uma produção de 110 a 120 t.ha $^{-1}$ de forragem.

Como forrageira, o fato de essa planta se armazenar naturalmente com pouca queda do seu valor nutritivo, que depende basicamente do seu teor de sacarose (BoIn et al., 1987; MoreIra, 1983; SAllas et al., 1992), é uma característica importante.

Nunes Junior (1987) mostra que as variedades de cana-de-açúcar apresentam curvas de maturação diferentes, sendo distintos, nessa curva, a porcentagem de sacarose e o florescimento. Esses resultados sugerem que, em uma atividade cujo período de colheita é muito longo, deveria ser utilizada mais de uma variedade, minimizando assim a perda de qualidade da forragem pelo corte antes do ponto de maturação (MATSUOKA e HoffMANN, 1993).

Lovadini et al. (1967) determinaram a composição química de 39 variedades de cana-deaçúcar forrageira. Os autores verificaram variação de $1,29 \%$ a $4,33 \%$ para o teor de proteína; $17,40 \%$ a $42,73 \%$ para a fibra bruta; $1,61 \%$ a $5,87 \%$ para o extrato etéreo; $0,75 \%$ a $4,65 \%$ para a matéria mineral e $51,20 \%$ a $76,69 \%$ para o teor de extrativos não nitrogenados.

BANDa e VAlDEZ (1976), estudando o efeito do estágio de maturidade sobre o valor nutritivo da canade-açúcar, observaram redução nos componentes da fibra de $61,10 \%$ para $54,10 \%$ para a fibra insolúvel em detergente neutro, de $37,7 \%$ para $33,40 \%$ para fibra insolúvel em detergente ácido, de $28,60 \%$ para $26,20 \%$ para a celulose e de $6,24 \%$ para $5,43 \%$ para a lignina, quando se analisaram canas com oito e 16 meses de desenvolvimento respectivamente. Os autores ainda verificaram que, ao aumentar a porcentagem dos componentes da fibra, havia redução linear da digestibilidade in vitro.

Kung Junior e Stanley (1982), estudando o efeito do estádio de maturação no valor nutritivo da cana-de-açúcar, observaram para cana colhida aos seis e 24 meses, redução de $68,30 \%$ para $52,60 \%$ para a fibra insolúvel em detergente neutro; de $41,50 \%$ para $34,20 \%$ para a fibra insolúvel em detergente ácido; de $26,90 \%$ para $18,40 \%$ para hemicelulose; de $30,90 \%$ para $24,50 \%$ para celulose, enquanto para a lignina observou-se aumento de $6,3 \%$ para $7,3 \%$ respectivamente. OliveIRA et al. (1996), em estudo com 16 variedades de cana-de-açúcar, observou que a porcentagem de fibra insolúvel em detergente neutro variou de $45,10 \%$ a $58,00 \%$ e o teor de fibra insolúvel em detergente ácido de $25,9 \%$ a $37,50 \%$ na matéria seca.

Carvalho (1992) verificou em cinco variedades de cana-de-açúcar que a concentração máxima de fibra insolúvel em detergente neutro ocorria próximo dos 241 dias de vegetação, havendo redução no teor à medida que avançava a idade de corte além daquela idade. Tendo em vista o exposto, definiu-se como objetivo experimental comparar, em duas idades de corte, 60 genótipos de cana-de-açúcar para uso na alimentação de ruminantes.

\section{MATERIAL E MÉTODOS}

O experimento foi realizado em 1999/2000, no Núcleo de Pesquisas Zootécnicas do Planalto Central, Instituto de Zootecnia, Nova Odessa (SP).

Foram estudados 60 genótipos de cana-deaçúcar, sendo 24 cultivares (SP70-1078, SP70-1143, SP71-799, SP71-1406, SP72-1861, SP79-1011, SP792233, SP79-2312, SP80-1842, IAC51-205, IAC52-150, IAC66-6, IAC68-12, IAC77-51, IAC82-2045, IAC823092, IAC86-2210, IAC87-3396, RB785750, RB806043, RB835486, RB855036, CB41-76 e CB45-3) e 36 clones (SP71-6180, SP71-6192, IAC80-1071, IAC80-3010, IAC80-3062, IAC81-1019, IAC81-1050, IAC81-2004, IAC81-3049, IAC82-1004, IAC82-3111, IAC82-3258, IAC83-1144, IAC83-1313, IAC83-2045, IAC83-2285, IAC83-2396, IAC83-2405, IAC83-4107, IAC83-4128, IAC83-4157, IAC83-4531, IAC84-1042, IAC85-1004, IAC85-3017, IAC85-3229, IAC86-1034, IAC86-1054, IAC86-1056, IAC86-1061, IAC87-1017, IAC87-1365, IAC87-1392, IAC87-3184, IAC87-3187 e IAC87-3420), fornecidos pelo Pólo Regional de Desenvolvimento Tecnológico dos Agronegócios do Centro-Sul, APTA, em Piracicaba (SP).

O terreno foi preparado com aplicação de calcário dolomítico, na base de $4.500 \mathrm{~kg} \cdot \mathrm{ha}^{-1}$ e o plantio, efetuado em março de 1998, aplicando-se no sulco 60 e $100 \mathrm{~kg}_{\text {. ha }}{ }^{-1}$ de $\mathrm{K}_{2} \mathrm{O}$ e $\mathrm{P}_{2} \mathrm{O}_{5}$ respectivamente.

Cada genótipo foi plantado em parcelas com 3,0 metros de comprimento, em quatro linhas de plantio, com espaçamento entre linhas de 1,0 metro. A distribuição das mudas no sulco foi contínua, colocando-se dois colmos na posição pé com ponta. Após a distribuição das mudas, os colmos foram cortados em toletes de aproximadamente três a quatro gemas. A cobertura foi efetuada colocando-se de 5 a $10 \mathrm{~cm}$ de terra sobre as mudas. 
O delineamento experimental foi em blocos casualizados, com três repetições, sendo os genótipos avaliados nas parcelas experimentais e as idades nas subparcelas, que correspondiam a uma das duas linhas centrais da parcela.

A amostragem, em cada subparcela, foi efetuada cortando-se um metro linear em uma das duas linhas centrais de cada parcela, sendo retirada uma amostra aos 12 e outra aos 18 meses de idade. As amostras para determinar as porcentagens de matéria seca $(\% \mathrm{MS})$, proteína bruta $(\% \mathrm{~PB})$, extrato etéreo (\%EE), matéria mineral (\%MM), fibra insolúvel em detergente neutro (\%FDN), fibra insolúvel em detergente ácido (\%FDA), celulose (\%CEL), hemicelulose (\%HEM), lignina (\%LIG) e carboidratos totais não estruturais (\%CTN), constituíram-se de duas canas, inteiras, que foram picadas em ensiladeira, regulada para porções de $3 \mathrm{~mm}$. Após a homogeneização de cada amostra, retirou-se uma porção que, após pesagem, foi colocada em estufa com circulação de ar, regulada para $65{ }^{\circ} \mathrm{C}$, onde permaneceu por 72 horas. Em seguida, foram novamente pesadas e moídas em moinho com peneira de $1 \mathrm{~mm}$. Após a moagem, as amostras foram analisadas conforme SiLva (1998) e GoERING e SoEsT van (1970). Para cada uma dessas características, efetuou-se análise de variância e aplicado os testes de Tukey e de Scott e KnotT (1974).

\section{RESULTADOS E DISCUSSÃO}

Pela tabela 1 observa-se a significância do teste $\mathrm{F}$ das variáveis estudadas. Pelos resultados, pode-se verificar que para \%MS, \%PB, \%EE e \%MM não foram observadas interações entre genótipos e idades de corte.

Tabela 1. Significância do teste F para as características estudadas

\begin{tabular}{lccc}
\hline Características & Genótipos $(\mathrm{G})$ & Idades de corte $(\mathrm{I})$ & Interação $(\mathrm{G} \times \mathrm{I})$ \\
\hline$\% \mathrm{MS}$ & $*$ & $*$ & $\mathrm{~ns}$ \\
$\% \mathrm{~PB}$ & $*$ & $\mathrm{~ns}$ & $\mathrm{~ns}$ \\
$\% \mathrm{EE}$ & $*$ & $*$ & $\mathrm{~ns}$ \\
$\% \mathrm{MM}$ & $*$ & $*$ & $\mathrm{~ns}$ \\
$\% \mathrm{CTN}$ & $*$ & $*$ & $*$ \\
$\% \mathrm{FDN}$ & $*$ & $*$ & $*$ \\
$\% \mathrm{FDA}$ & $*$ & $*$ & $*$ \\
$\% \mathrm{CEL}$ & $*$ & $*$ & $*$ \\
$\% \mathrm{LIG}$ & $*$ & $*$ & $*$ \\
\hline
\end{tabular}

* = Significantivo a $5 \%$ de probabilidade. $n s$ = Não significantivo.

Na tabela 2 são apresentadas as porcentagens, média do corte de 12 e 18 meses, MS, PB, EE, e MM determinadas nos genótipos, bem como as médias gerais, e os coeficientes de variação.

Comparando as \%MS entre as idades estudadas, verificou-se que os valores para os genótipos colhidos aos 12 meses $(30,60 \%)$ foi maior do que aqueles dos genótipos colhidos aos 18 meses $(25,88 \%)$. Esse resultado pode ser explicado pelo longo período seco, ocorrido até novembro, após o corte dos genótipos aos 12 meses; esses teores de MS são próximos de $30 \%$ encontrado na literatura (BoIn et al. 1987). Os 60 genótipos formaram três grupos, quanto ao teor de MS. Os grupos 1, 2 e 3 foram formados por 9, 21 e 30 genótipos, respectivamente, mostrando que mais da metade dos genótipos apresentaram teores acima de $25 \%$ de MS.

O teor de $\mathrm{PB}$ dos genótipos cortados aos 12 meses $(2,59 \%)$ foi maior que aquele dos genótipos cortados aos 18 meses $(2,34 \%)$. Essa pequena diferença provavelmente pode ser explicada pela maior quantidade de matéria seca de ponta (folhas) na cana mais nova. Esses teores estão dentro da amplitude de variação $(1,29 \%$ a $4,33 \%)$ obtida por LovADINI et al. (1967) para 39 variedades de cana. Na média das idades, formaram-se dois grupos quanto ao teor de PB: um de 32 genótipos, com limite superior de 2,34, e outro de 28 genótipos, com valor máximo de 3,20. 
Tabela 2. Porcentagens, de matéria seca (\%MS), proteína bruta (\%PB), extrato etéreo (\%EE) e matéria mineral (\%MM), dos genótipos de cana-de-açúcar, média dos corte de 12 e 18 meses de idade

\begin{tabular}{|c|c|c|c|c|}
\hline Cultivar & $\%$ MS & $\% \mathrm{~PB}$ & $\% \mathrm{EE}$ & $\% \mathrm{MM}$ \\
\hline IAC52-150 & 32,54 a3 & 2,17 a1 & 0,68 a 1 & 2,10 a1 \\
\hline IAC 83-2405 & 32,05 a3 & 2,78 a2 & 1,31 a2 & 3,00 a2 \\
\hline SP70-1078 & 31,20 a3 & 2,54 a2 & 1,54 a3 & 2,64 a2 \\
\hline IAC $87-3184$ & 30,86 a3 & 2,43 a1 & 0,90 a1 & 2,43 a 1 \\
\hline SP70-1143 & 30,32 a3 & 2,26 a1 & 1,21 a2 & 2,21 a 1 \\
\hline RB83-5486 & 30,31 a3 & 2,47 a1 & 1,04 a1 & 2,35 a1 \\
\hline IAC 81-1019 & 30,27 a3 & 2,88 a2 & 0,94 a1 & 2,67 a2 \\
\hline RB80-6043 & 30,08 a3 & 2,36 a1 & 0,86 a1 & 2,37 a1 \\
\hline IAC 83-2285 & 29,84 a3 & 2,78 a2 & 1,49 a3 & 3,03 a2 \\
\hline SP80-1842 & 29,80 a3 & 2,62 a2 & 1,22 a2 & 2,54 a1 \\
\hline IAC $80-1071$ & 29,64 a3 & 2,64 a2 & 0,92 a1 & 2,65 a2 \\
\hline IAC 86-1054 & 29,63 a3 & 2,60 a 2 & 0,92 a 1 & 2,80 a2 \\
\hline RB78-5750 & 29,58 a3 & 2,58 a2 & 1,00 a1 & 2,83 a2 \\
\hline IAC $80-3062$ & 29,38 a3 & 1,99 a1 & 0,75 a1 & 2,37 a 1 \\
\hline RB85-5036 & 29,36 a3 & 2,04 a1 & 0,94 a1 & 2,14 a1 \\
\hline IAC 86-1061 & 29,32 a3 & 2,31 a1 & 1,10 a2 & 2,45 a1 \\
\hline IAC $81-3049$ & 29,31 a3 & 2,32 a1 & 1,01 a1 & 2,52 a1 \\
\hline IAC 83-4128 & 29,10 a3 & 2,98 a2 & 1,13 a2 & 3,03 a2 \\
\hline IAC 83-2396 & 29,05 a3 & 1,91 a1 & 1,04 a1 & 2,39 a 1 \\
\hline IAC 87-1365 & 29,03 a3 & 2,37 a1 & 1,00 a1 & 2,36 a1 \\
\hline IAC66-6 & 29,01 a3 & 2,36 a1 & 0,80 a 1 & 2,78 a2 \\
\hline SP71-6180 & 28,92 a3 & 2,10 a1 & 0,79 a1 & 2,68 a 1 \\
\hline IAC 82-3111 & 28,91 a3 & 2,56 a2 & 1,15 a2 & 2,58 a2 \\
\hline IAC 85-3229 & 28,63 a3 & 2,59 a2 & 0,89 a1 & 2,34 a1 \\
\hline IAC 86-1056 & 28,62 a3 & 2,25 a1 & 1,04 a1 & 2,17 a1 \\
\hline IAC51-205 & 28,54 a3 & 2,43 a1 & 1,23 a2 & 2,60 a2 \\
\hline CB45-3 & 28,48 a3 & 2,14 a1 & 0,76 a1 & 2,69 a2 \\
\hline SP79-1011 & 28,42 a3 & 1,92 a1 & 0,68 a1 & 2,03 a 1 \\
\hline SP71-799 & 28,30 a3 & 2,68 a2 & 0,92 a 1 & 2,79 a2 \\
\hline IAC 82-1004 & 28,29 a3 & 3,20 a2 & 0,93 a 1 & 2,92 a2 \\
\hline IAC 83-4107 & 28,20 a2 & 2,30 a1 & 1,48 a3 & 2,47 a 1 \\
\hline IAC 87-3396 & 28,17 a2 & 2,33 a1 & 1,19 a2 & 3,28 a2 \\
\hline SP71-6192 & 28,09 a2 & 2,19 a1 & 0,85 a1 & 2,37 a1 \\
\hline IAC 83-1144 & 28,08 a2 & 2,33 a1 & 0,93 a1 & 2,73 a2 \\
\hline IAC 87-3187 & 27,95 a2 & 2,82 a2 & 1,07 a2 & 2,64 a2 \\
\hline IAC68-12 & 27,88 a2 & 2,96 a2 & 0,73 a1 & 2,33 a 1 \\
\hline IAC 86-1034 & 27,86 a2 & 2,23 a1 & 1,06 a2 & 2,50 a1 \\
\hline IAC 85-3017 & 27,84 a2 & 2,21 a1 & 1,03 a1 & 2,47 a1 \\
\hline IAC 82-3258 & 27,84 a2 & 2,59 a2 & 1,00 a 1 & 2,45 a1 \\
\hline IAC 87-1392 & 27,77 a2 & 2,81 a2 & 0,86 a 1 & 2,93 a2 \\
\hline CB41-76 & 27,67 a2 & 2,89 a2 & 0,96 a1 & 3,00 a2 \\
\hline IAC $83-4531$ & 27,62 a2 & 2,37 a1 & 0,69 a1 & 2,43 a1 \\
\hline IAC77-51 & 27,59 a2 & 2,08 a1 & 0,89 a1 & 2,51 a1 \\
\hline IAC 85-1004 & 27,58 a2 & 2,58 a2 & 0,64 a1 & 2,72 a2 \\
\hline IAC 82-3092 & 27,42 a2 & 2,32 a1 & 1,01 a1 & 2,51 a 1 \\
\hline IAC 86-2210 & 27,27 a2 & 2,74 a2 & 0,81 a1 & 2,06 a1 \\
\hline IAC $84-1042$ & 27,04 a 2 & 2,55 a2 & 1,08 a2 & 3,27 a2 \\
\hline IAC 83-2045 & 26,99 a2 & 3,08 a2 & 0,96 a 1 & 2,45 a1 \\
\hline IAC 80-3010 & 26,81 a 2 & 2,61 a2 & 0,79 a1 & 2,87 a2 \\
\hline IAC 82-2045 & 26,81 a2 & 2,43 a1 & 0,98 a1 & 2,67 a2 \\
\hline IAC 87-420 & 26,59 a2 & 2,01 a1 & 0,71 a1 & 2,07 a1 \\
\hline IAC 81-1050 & 26,28 a1 & 2,55 a2 & 0,88 a1 & 2,65 a2 \\
\hline SP71-1406 & 26,14 a 1 & 2,53 a 2 & 1,24 a2 & 2,37 a1 \\
\hline SP72-1861 & 26,11 a 1 & 2,43 a1 & 1,08 a2 & 2,66 a2 \\
\hline IAC 81-2004 & 25,83 a1 & 1,91 a1 & 0,84 a1 & 2,07 a1 \\
\hline SP79-2233 & 25,70 a1 & 2,83 a2 & 1,01 a1 & 2,55 a1 \\
\hline IAC 83-4157 & 25,47 a1 & 2,42 a1 & 1,18 a 2 & 2,81 a2 \\
\hline SP79-2312 & 24,73 a1 & 2,69 a2 & 0,79 a1 & 2,32 a 1 \\
\hline IAC $83-1313$ & 24,63 a1 & 2,17 a1 & 0,83 a1 & 2,17 a1 \\
\hline IAC 87-1017 & 23,66 a1 & 2,62 a2 & 0,81 a 1 & 2,35 a1 \\
\hline Média & 28,24 & 2,46 & 0,98 & 2,55 \\
\hline CV (\%) genótipo & 7,60 & 16,32 & 24,37 & 19,79 \\
\hline CV (\%) idade & 8,54 & 15,90 & 26,06 & 15,45 \\
\hline
\end{tabular}

Médias seguidas de letras e números distintos, na coluna, diferem pelo teste de Scott-Knott $(1974)$, com $(P<0,05)$. 
Quanto ao teor de EE, foram separados três grupos distintos, formados por 43, 14 e três genótipos. De maneira geral, os teores de EE foram menores que aqueles determinados por LovADINI et al (1967), que observaram variação de $1,61 \%$ a $5,87 \%$ em 39 variedades de cana forrageira. Ressalta-se que parte do extrato etéreo da cana-de-açúcar pode ser originária das ceras que recobrem os colmos, as quais são de baixa utilização pelos ruminantes (Boin et al., 1987).

O teor de MM nos genótipos cortados aos 18 meses $(2,79 \%)$ foi maior que aquele da idade de 12 meses $(2,32 \%)$. Os genótipos foram agrupados em dois grupos distintos, um com 34 e outro com 26 genótipos. Os teores de MM (2,03\% a 3,28\%) foram menores que aqueles observados por LovADINI et al. (1967).

$\mathrm{Na}$ Tabela 3 são mostradas as porcentagens de carboidratos totais não estruturais (\%CTN), fibra insolúvel em detergente neutro $(\% \mathrm{FDN})$ e fibra insolúvel em detergente ácido (\%FDA) dos genótipos colhidos aos 12 e 18 meses de idade, bem como as médias e os coeficientes de variação.

Observaram-se nos genótipos SP71-799, SP792233, SP80-1842, IAC52-150, IAC66-6, IAC68-12, IAC77-51, IAC80-1071, IAC80-3010, IAC81-1050, IAC81-2004, IAC82-1004, IAC82-2045, IAC83-1313, IAC83-2396, IAC84-1042, IAC86-1054, IAC87-3420 e RB855036 teores de CTN maiores quando cortados com 12 meses de idade, enquanto em IAC83-2405 e IAC87-1017, o teor de CTN foi maior quando o corte ocorreu aos 18 meses. Nos demais genótipos, os teores de CTN foram semelhantes nas duas idades de corte. Esses resultados mostraram que os genótipos apresentaram curvas de maturidade diferentes, conforme relata NunES JUNIOR (1987) e MATSUOKA e Hoffmann (1993). Ainda quanto ao teor de CTN, os 60 genótipos se agruparam em 15 e 12 grupos diferentes nas idades de 12 e 18 meses respectivamente.

No geral, os teores de CTN determinados nos genótipos colhidos aos 18 meses foram menores que aqueles da colheita aos 12 meses. Aos 18 meses, somente 22 genótipos apresentaram teores de CTN acima da média geral, enquanto na idade de 12 meses esse número foi de 43 genótipos.

Essa característica é muito importante para selecionar cultivares para alimentação animal, pois a digestibilidade da matéria seca é positivamente correlacionada com o teor de sacarose (BoIn et al., 1987). Por outro lado, segundo MATsUOKA e HoffmAnN (1993), em sistemas nos quais a cana-de-açúcar é utilizada por um período muito grande do ano, devese dar preferência para o uso de dois ou mais cultivares com maturação diferentes, sugerindo a utilização de genótipos dos grupos que mostraram maior teor de CTN na idade de 12 e 18 meses.

Quanto aos teores de FDN, nos genótipos SP71-799, SP71-6192, SP79-2233, IAC52-150, IAC66-6, IAC80-3010, IAC81-1050, IAC81-2004, IAC81-3049, IAC82-1004, IAC82-2045, IAC83-1313, IAC83-2396, IAC83-4128, IAC86-1034, IAC87- 3420 e RB855036 verificaram teores maiores na idade de 18 meses, enquanto nos demais, os teores de FDN foram semelhantes nas duas idades de corte. Essa resposta foi contrária à observada por BANDA e VALDEZ (1976), Kung Jr. e Stanley (1982), Carvalho (1992) e Oliveira (1996), que verificaram decréscimo no teor de FDN à medida que a cana-de-açúcar era colhida em idade mais avançada. Por outro lado, pode-se observar que na maioria dos genótipos com teores de FDN maiores na idade de 18 meses, os teores de CTN foram menores nessa idade, sugerindo que nesses genótipos com pico de maturação aos 12 meses, há perda de CTN e, em conseqüência, aumento relativo dos teores de FDN.

Pelos resultados, pode-se dizer que os teores de FDN dos genótipos colhidos na idade de 18 meses foram maiores que aqueles verificados aos 12 meses. Observou-se também que, embora não tenha sido efetuada análise de correlação, os grupos que apresentaram maior teor de CTN mostraram menores teores de FDN (Tabela 3). Ainda com referência ao teor de FDN, os 60 genótipos foram agrupados em 11 e 10 grupos diferentes nas idades de 12 e 18 meses respectivamente.

Esses resultados podem revelar que os genótipos com menor teor de FDN teriam maior digestibilidade da matéria seca, e aqueles com maiores teores de sacarose, também mostram maior digestibilidade da matéria seca (Born et al., 1987).

Em relação ao teor de FDA, nos genótipos SP71-1406, SP71-6192, SP79-2233, IAC80-3010, IAC811050, IAC81-2004, IAC81-3049, IAC82-1004, IAC82-2045, IAC83-2396, IAC83-4128, IAC83-4157, IAC84-1042, IAC86-1034, IAC87-3396, IAC87-3420 e RB855036 verificaram-se teores maiores na idade de 18 meses, enquanto em IAC86-1056 o teor de FDA foi menor aos 18 meses.

Os demais genótipos apresentaram teores de FDA semelhantes nas duas idades de corte. Em relação aos genótipos que apresentaram teor igual de FDN nas duas idades, apenas SP71-1406, IAC83-4157, IAC841042 e IAC87-3396 apresentaram teores de FDA maior na idade de 18 meses, podendo sugerir que, nesses, o teor de fibra foi maior em virtude do aumento de celulose e lignina. Nos demais, em que tanto \%FDN como \%FDA aumentaram com a idade, o teor de hemicelulose pode ter sido maior. 
Tabela 3. Porcentagens de carboidratos não estruturais (\%CTN) fibra insolúvel em detergente neutro (\%FDN), e fibra insolúvel em detergente (\%FDA), nos genótipos de cana-de-açúcar colhidos aos 12 e 18 meses de idade

\begin{tabular}{|c|c|c|c|c|c|c|}
\hline \multirow{2}{*}{ Cultivar } & \multicolumn{2}{|c|}{$\% \mathrm{CTN}$} & \multicolumn{2}{|c|}{$\%$ FDN } & \multicolumn{2}{|c|}{$\%$ FDA } \\
\hline & 12 meses & 18 meses & 12 meses & 18 meses & 12 meses & 18 meses \\
\hline RB85-5036 & 65,12 a15 A2 & 47,24 a10 A1 & 37,03 a1 A1 & 52,14 a5 A2 & 23,46 a3 A1 & 30,44 a5 A2 \\
\hline IAC $83-2396$ & 59,47 a14 A2 & 44,12 a9 A1 & 39,33 a2 A1 & 52,72 a5 A2 & 23,18 a3 A1 & 30,84 a 6 A2 \\
\hline IAC77-51 & 58,54 a14 A2 & 36,68 a5 A1 & 41,01 a3 A1 & 44,91 a1 A1 & 26,52 a7 A1 & 28,27 a3 A1 \\
\hline IAC68-12 & 58,08 a14 A2 & 44,59 a 9 A1 & 43,49 a 4 A1 & 44,06 a1 A1 & 29,84 a11 A1 & 27,79 a3 $\mathrm{A} 1$ \\
\hline IAC 83-1313 & 55,93 a13 A2 & 44,69 a9 A1 & 37,33 a1 A1 & 48,56 a3 A2 & 22.90 a3 A1 & 27,72 a3 A1 \\
\hline IAC 85-1004 & 55,91 a 13 A1 & 47,07 a10 A1 & 39,35 a 2 A1 & 47,19 a 2 A1 & 23,74 a 4 A1 & 27,26 a2 A1 \\
\hline SP79-1011 & 54,71 a13 A1 & 48,59 a10 A1 & 41,83 a3 A1 & 44,68 a1 A1 & 24,22 a 4 A1 & 26,27 a1 A1 \\
\hline IAC $82-1004$ & 52,90 a12 A2 & 32,67 a 3 A 1 & 41,05 a 3 A1 & 54,82 a7 A2 & 25,26 a5 A1 & 32,86 a7 A2 \\
\hline IAC 82-3258 & 52,80 a12 A1 & 46,46 a10 A1 & 41,48 a3 A1 & 48,63 a3 A1 & 23,91 a 4 A1 & 28,11 a3 A1 \\
\hline IAC $80-1071$ & 51,91 a 12 A2 & 34,74 a 3 A1 & 46,22 a5 A1 & 49,19 a3 A1 & 29,21 a 10 A 1 & 31,95 a 6 A1 \\
\hline IAC 81-2004 & 51,75 a12 A2 & 32,32 a3 A1 & 42,83 a 4 A1 & 52,17 a5 A2 & 25,22 a5 A1 & 31,40 a6 A2 \\
\hline IAC 86-1034 & 51,32 a11 $\mathrm{A} 1$ & 41,57 a7 A1 & 45,67 a5 A1 & 54,24 a 6 A2 & 25,25 a5 A1 & 31,79 a6 A2 \\
\hline IAC $87-3420$ & 51,29 a11 A2 & 38,94 a 6 A1 & 35,79 a1 A1 & 55,77 a7 A2 & 21,14 a1 A1 & 31,51 a6 A2 \\
\hline SP80-1842 & 50,98 a11 A2 & 36,36 a4 A1 & 44,01 a4 A1 & 50,05 a4 A1 & 27,13 a7 A1 & 30,83 a 6 A1 \\
\hline IAC52-150 & 50,51 a11 A2 & 33,08 a3 A1 & 42,12 a3 A1 & 51,87 a5 A2 & 26,23 a6 A1 & 31,19 a6 A1 \\
\hline IAC $81-1050$ & 50,28 a11 A2 & 32,03 a3 A1 & 40,67 a3 A1 & 54,35 a 6 A2 & 22,63 a2 A1 & 32,35 a7 A2 \\
\hline SP71-799 & 50,16 a11 A2 & 33,80 a3 A1 & 45,97 a5 A1 & 54,69 a7 A2 & 27,32 a7 A1 & 31,58 a 6 A1 \\
\hline IAC $87-3396$ & 49,26 a10 A1 & 43,53 a9 A1 & 46,08 a5 A1 & 54,11 a6 A1 & 26,10 a6 A1 & 32,07 a 6 A2 \\
\hline IAC66-6 & 49,17 a10 A2 & 33,65 a3 A1 & 40,20 a3 A1 & 53,25 a5 A2 & 29,05 a10 A1 & 31,27 a 6 A1 \\
\hline IAC $84-1042$ & 48,32 a10 A2 & 37,01 a5 A1 & 46,70 a 6 A1 & 52,36 a5 A1 & 26,12 a 6 A1 & 31,17 a6 A2 \\
\hline IAC $80-3062$ & 47,66 a10 A1 & 53,85 a12 A1 & 47,28 a 6 A1 & 46,72 a 2 A1 & 28,88 a9 A1 & 27,32 a2 $A 1$ \\
\hline SP71- 6192 & 47,63 a10 A1 & 40,31 a7 A1 & 36,89 a1 A1 & 48,40 a3 A2 & 21,85 a1 A1 & 27,73 a3 A2 \\
\hline CB $41-76$ & 46,49 a9 A1 & 41,20 a7 A1 & 48,19 a7 A1 & 52,45 a5 A1 & 27,93 a 8 A1 & 29,44 a 4 A1 \\
\hline IAC 85-3017 & 46,33 a9 A1 & 42,21 a 8 A1 & 47,94 a7 A1 & 48,03 a3 A1 & 29,25 a10 A1 & 29,77 a 4 A1 \\
\hline IAC $83-2405$ & 46,01 a9 A2 & 33,51 a3 A1 & 47,84 a7 $\mathrm{A} 1$ & 53,96 a 6 A1 & 28,55 a 9 A 1 & 33,34 a8 $A 1$ \\
\hline IAC 82-3111 & 45,68 a9 A1 & 38,89 a 6 A1 & 51,57 a9 A1 & 57,53 a 8 A1 & 30,60 a11 A1 & 33,46 a 8 A1 \\
\hline SP79-2312 & 45,47 a 9 A1 & 44,83 a9 $\mathrm{A} 1$ & 43,14 a 4 A1 & 48,60 a3 A1 & 25,64 a5 A1 & 28,99 a 4 A 1 \\
\hline SP71-6180 & 45,44 a9 A1 & 35,94 a4 A1 & 46,08 a5 A1 & 53,97 a 6 A1 & 27,81 a 8 A1 & 31,88 a 6 A1 \\
\hline SP79-2233 & 45,26 a9 A2 & 29,54 a2 A1 & 44,41 a 4 A1 & 55,78 a7 A2 & 25,49 a5 A1 & 32,81 a7 A2 \\
\hline RB78-5750 & 44,80 a9 A1 & 39,19 a 6 A1 & 47,42 a 6 A1 & 54,91 a7 A1 & 27,77 a 8 A1 & 32,69 a7 A1 \\
\hline SP72-1861 & 44,62 a 8 A1 & 43,34 a9 A1 & 49,09 a 8 A1 & 53,31 a5 A1 & 27,75 a8 A1 & 30,98 a 6 A1 \\
\hline RB80-6043 & 44,09 a 8 A1 & 37,40 a5 A1 & 48,18 a7 A1 & 48,94 a3 A1 & 27,78 a 8 A1 & 27,82 a3 A1 \\
\hline CB45-3 & 44,02 a8 A1 & 35,22 a4 A1 & 46,37 a 6 A1 & 50,65 a4 A1 & 29,24 a10 A1 & 29,22 a 4 A1 \\
\hline IAC $81-3049$ & 43,65 a 8 A 1 & 37,45 a5 A1 & 46,98 a 6 A1 & 57,05 a 8 A2 & 27,96 a 8 A 1 & 33,24 a8 A2 \\
\hline IAC $80-3010$ & 43,10 a8 A2 & 26,49 a1 A1 & 49,50 a 8 A1 & 59,27 a9 A2 & 30,04 a11 A1 & 36,89 a10 A2 \\
\hline IAC $83-4157$ & 42,81 a 8 A1 & 34,03 a3 A1 & 45,19 a5 A1 & 48,82 a3 A1 & 25,25 a5 A1 & 30,53 a5 A2 \\
\hline IAC 86-1054 & 42,31 a7 A2 & 30,43 a2 A1 & 48,31 a7 A1 & 54,78 a7 A1 & 29,35 a10 A1 & 32,65 a7 A1 \\
\hline IAC $83-4531$ & 42,05 a7 A1 & 33,09 a3 A1 & 50,03 a 8 A1 & 51,14 a 4 A1 & 28,80 a 9 A 1 & 29,48 a 4 A1 \\
\hline IAC51-205 & 41,87 a7 A1 & 36,59 a5 A1 & 50,25 a 8 A1 & 49,26 a3 A1 & 29,23 a10 A1 & 30,33 a5 A1 \\
\hline SP70-1078 & 41,71 a7 $\mathrm{A} 1$ & 33,60 a3 $\mathrm{A} 1$ & 55,88 a11 A1 & 57,75 a 8 A 1 & 32,01 a12 A1 & 33,59 a 8 A1 \\
\hline IAC 85-3229 & 41,30 a7 A1 & 39,52 a 6 A1 & 44,32 a 4 A1 & 52,29 a5 A1 & 26,77 a7 A1 & 30,55 a5 A1 \\
\hline IAC $87-3187$ & 40,62 a7 A1 & 43,74 a 9 A1 & 51,19 a 9 A1 & 51,42 a 4 A1 & 30,13 a $11 \mathrm{~A} 1$ & 31,04 a 6 A1 \\
\hline IAC 87-1365 & 40,38 a7 A1 & 42,38 a 8 A1 & 45,87 a5 A1 & 49,58 a3 A1 & 26,64 a7 A1 & 28,87 a 4 A 1 \\
\hline SP70-1143 & 40,12 a 6 A1 & 39,39 a6 A1 & 48,37 a7 A1 & 51,93 a5 A1 & 28,52 a9 A1 & 31,43 a 6 A1 \\
\hline RB83-5486 & 40,02 a 6 A1 & 40,46 a7 A1 & 45,07 a5 A1 & 51,04 a 4 A1 & 27,35 a7 A1 & 29,64 a 4 A1 \\
\hline IAC 83-4107 & 39,97 a6 A1 & 42,00 a 8 A1 & 45,64 a5 A1 & 48,89 a3 A1 & 26,76 a7 A1 & 28,73 a3 A1 \\
\hline IAC 86-2210 & 39,82 a 6 A1 & 37,59 a5 A1 & 48,42 a7 A1 & 48,85 a3 A1 & 27,18 a7 A1 & 28,43 a3 A1 \\
\hline IAC 83-1144 & 39,55 a6 A1 & 39,09 a6 A1 & 52,11 a9 A1 & 53,51 a6 A1 & 32,42 a12 A1 & 31,45 a 6 A1 \\
\hline IAC $82-2045$ & 39,10 a 6 A2 & 27,57 a1 A1 & 45,18 a5 A1 & 65,73 a10 A2 & 26,10 a 6 A1 & 36,87 a10 A2 \\
\hline IAC $87-3184$ & 39,00 a6 A1 & 31,31 a2 $\mathrm{A} 1$ & 46,61 a6 A1 & 52,05 a5 A1 & 29,19 a10 A1 & 31,13 a6 A1 \\
\hline IAC 82-3092 & 38,82 a 6 A1 & 33,74 a3 A1 & 49,19 a 8 A1 & 52,35 a 5 A 1 & 34,66 a13 A1 & 31,12 a 6 A1 \\
\hline IAC 86-1061 & 38,61 a6 A1 & 37,16 a5 A1 & 47,50 a7 A1 & 52,38 a5 A1 & 27,84 a 8 A1 & 30,37 a5 A1 \\
\hline IAC 83-2045 & 38,32 a 6 A1 & 51,04 a11 A2 & 50,54 a9 A1 & 49,63 a3 A1 & 30,37 a11 A1 & 29,38 a 4 A1 \\
\hline IAC 86-1056 & 37,25 a 6 A1 & 43,74 a9 A1 & 48,96 a 8 A1 & 51,88 a5 A1 & 35,55 a14 A2 & 30,26 a5 A1 \\
\hline IAC $83-4128$ & 36,42 a5 A1 & 29,51 a 2 A1 & 48,24 a7 A1 & 58,91 a9 A2 & 29,12 a 10 A1 & 34,99 a9 A2 \\
\hline IAC 87-1017 & 34,88 a5 A1 & 46,44 a10 A2 & 49,64 a 8 A1 & 49,34 a3 A1 & 30,04 a11 A1 & 27,95 a3 A1 \\
\hline IAC 81-1019 & 33,00 a4 A1 & 34,95 a3 A1 & 53,44 a10 A1 & 53,03 a5 A1 & 30,40 a11 A1 & 32,29 a7 A1 \\
\hline SP71-1406 & 31,02 a3 A1 & 35,22 a4 A1 & 49,54 a 8 A1 & 51,79 a5 A1 & 24,52 a 4 A1 & 30,60 a5 A2 \\
\hline IAC $87-1392$ & 28,80 a2 A1 & 37,81 a5 A1 & 54,91 a11 A1 & 53,80 a 6 A 1 & 31,50 a12 A1 & 30,77 a 6 A1 \\
\hline IAC $83-2285$ & 26,59 a1 A1 & 25,56 a 1 A 1 & 56,25 a11 A1 & 57,46 a 8 A1 & 32,64 a12 A1 & 36,20 a10 A1 \\
\hline Média & \multicolumn{2}{|c|}{41,65} & \multicolumn{2}{|c|}{49,14} & \multicolumn{2}{|c|}{29,24} \\
\hline CV (\%) genótipo & \multicolumn{2}{|c|}{15,00} & \multicolumn{2}{|c|}{8,43} & \multicolumn{2}{|c|}{9,38} \\
\hline $\mathrm{CV}(\%)$ idade & \multicolumn{2}{|c|}{15,03} & \multicolumn{2}{|c|}{10,22} & \multicolumn{2}{|c|}{10,56} \\
\hline
\end{tabular}

Médias seguidas de letras minúsculas e números distintos, na coluna, diferem pelo teste de Scott-Knott $(1974)$, com $(\mathrm{P}<5 \%)$; Médias seguidas de letras maiúsculas e números distintos, na linha, diferem pelo Teste de Tukey, com $(P<0,05)$. 
A resposta dos genótipos com os maiores teores de FDA na idade de 18 meses foi contrária à encontrada por BANDA e VAldez (1976), Kung Jr. e Stanley (1982), Carvalho (1992) e Oliveira (1996), que determinaram a composição de cana-de-açúcar de 6 aos 24 meses, verificando decréscimo no teor de FDA, à medida que a cana-de-açúcar era colhida em idade mais avançada.

Da mesma forma em que se observou para a FDN, também o teor de FDA foi, de modo geral, maior na idade de 18 meses (Tabela 3). Os 60 genótipos foram agrupados em 14 e 10 grupos diferentes nas idades de 12 e 18 meses, respectivamente, podendo sugerir que os genótipos pertencentes aos grupos com menores teores de FDA, nas idades de 12 e 18 meses, possivelmente teriam maior digestibilidade da matéria seca, posto que o teor de FDA é positivamente correlacionado com o teor de FDN.

$\mathrm{Na}$ Tabela 4 são apresentadas as \%CEL, \%HEM e \%LIG dos genótipos nas idades de 12 e 18 meses, assim como as médias e os coeficientes de variação.

Tabela 4. Porcentagens de celulose (\%CEL), hemicelulose (\%HEM) e lignina (\%LIG), nos genótipos de cana-de-açúcar colhidos aos 12 e 18 meses de idade

\begin{tabular}{|c|c|c|c|c|c|c|}
\hline \multirow{2}{*}{ Cultivar } & \multicolumn{2}{|c|}{$\%$ CEL } & \multicolumn{2}{|c|}{ \%HEM } & \multicolumn{2}{|c|}{$\%$ LIG } \\
\hline & 12 meses & 18 meses & 12 meses & 18 meses & 12 meses & 18 meses \\
\hline RB85-5036 & 18,06 a $1 \mathrm{~A} 1$ & 24,56 a3 A2 & 13,57 a 2 A1 & 21,71 a 6 A2 & 3,49 a 4 A1 & 4,75 a8 A2 \\
\hline IAC83-2396 & 21,15 a5 $\mathrm{A} 1$ & 25,97 a5 A2 & 16,15 a 4 A1 & 21,88 a7 A2 & 3,60 a5 A1 & 4,25 a5 A1 \\
\hline IAC77-51 & 22,06 a7 A1 & 23,77 a2 A1 & 14,50 a3 A1 & 16,64 a1 A1 & 4,04 a7 A1 & 4,23 a5 A1 \\
\hline IAC68-2 & 24,99 a11 A1 & 23,00 a1 A1 & 13,65 a 2 A1 & 16,27 a1 A1 & 4,39 a8 A1 & 4,33 a 6 A1 \\
\hline IAC 83-1313 & 19,31 a3 A1 & 24,08 a2 A2 & 14,44 a3 A1 & 20,84 a5 A2 & 2,71 a1 A1 & 3,61 a2 A1 \\
\hline IAC 85-1004 & 20,63 a 5 A1 & 23,44 a1 A1 & 15,61 a 4 A1 & 20,06 a5 A2 & 2,97 a2 A1 & 3,55 a2 A1 \\
\hline SP79-011 & 21,04 a5 $\mathrm{A} 1$ & 22,96 a1 A1 & 17,61 a 5 A1 & 18,41 a3 $\mathrm{A} 1$ & 2,85 a1 A1 & 3,33 a1 A1 \\
\hline IAC 82-1004 & 21,16 a5 A1 & 26,41 a5 A2 & 15,78 a 4 A1 & 21,96 a7 A2 & 3,54 a 4 A1 & 4,97 a10 A2 \\
\hline IAC $82-3258$ & 20,36 a 4 A1 & 23,17 a1 A1 & 17,57 a5 A1 & 20,52 a5 $\mathrm{A} 1$ & 3,01 a 2 A 1 & 4,10 a 4 A2 \\
\hline IAC 80-1071 & 24,80 a11 A1 & 26,78 a6 A1 & 17,01 a5 $\mathrm{A} 1$ & 17,24 a 2 A1 & 3,72 a5 $\mathrm{A} 1$ & 4,51 a7 A1 \\
\hline IAC 81-2004 & 18,53 a 2 A1 & 26,42 a5 A2 & 17,61 a5 $\mathrm{A} 1$ & 20,77 a5 A1 & 2,98 a 2 A 1 & 4,34 a6 A2 \\
\hline IAC $86-1034$ & 21,93 a7 A1 & 26,15 a5 A2 & 20,42 a 8 A1 & 22,44 a7 A1 & 3,09 a3 A1 & 4,43 a6 A2 \\
\hline IAC $87-3420$ & 17,94 a 1 A1 & 26,61 a 6 A2 & 14,65 a 3 A 1 & 22,92 a 8 A2 & 2,93 a2 A1 & 4,49 a7 A2 \\
\hline SP80-1842 & 23,18 a 9 A1 & 25,83 a5 A1 & 16,89 a5 $\mathrm{A} 1$ & 19,23 a 4 A1 & 3,64 a5 A1 & 4,71 a 8 A2 \\
\hline IAC52-150 & 21,97 a7 A1 & 25,00 a4 A1 & 15,89 a 4 A1 & 20,68 a5 A2 & 3,69 a5 A1 & 5,07 a10 A2 \\
\hline IAC $81-1050$ & 19,60 a3 A1 & 25,87 a5 A2 & 18,04 a5 $\mathrm{A} 1$ & 22,00 a7 A1 & 3,04 a 2 A1 & 5,59 a13 A2 \\
\hline SP71-799 & 23,28 a 9 A 1 & 26,31 a5 A1 & 18,65 a 6 A1 & 22,94 a 8 A1 & 3,61 a5 A1 & 4,45 a 6 A1 \\
\hline IAC 87-3396 & 22,22 a7 A1 & 25,43 a 4 A1 & 19,97 a 8 A 1 & 22,04 a7 A1 & 3,64 a5 A1 & 5,21 a11 A2 \\
\hline IAC66-6 & 24,41 a11 A1 & 25,96 a5 A1 & 11,15 a1 A1 & 21,98 a7 A2 & 4,40 a8 A1 & 4,87 a9 A1 \\
\hline IAC $84-1042$ & 22,00 a7 A1 & 25,28 a 4 A 1 & 20,58 a 8 A1 & 20,85 a5 A1 & 3,46 a4 A1 & 4,71 a8 $\mathrm{A} 2$ \\
\hline IAC 80-3062 & 26,13 a12 A1 & 22,93 a1 A1 & 18,40 a 6 A1 & 19,40 a 4 A1 & 3,68 a 5 A 1 & 3,63 a2 A1 \\
\hline SP71- 6192 & 18,89 a 2 A1 & 22,90 a1 A2 & 15,04 a3 A1 & 20,67 a5 A2 & 2,76 a 1 A 1 & 4,00 a4 A2 \\
\hline CB41-76 & 24,05 a10 A1 & 25,12 a 4 A1 & 20,26 a 8 A1 & 23,01 a 8 A1 & 3,83 a 6 A1 & 4,39 a6 A1 \\
\hline IAC 85-3017 & 24,59 a11 A1 & 24,37 a3 A1 & 18,69 a 6 A1 & 18,26 a3 A1 & 4,19 a7 A1 & 4,82 a9 A1 \\
\hline IAC 83-2405 & 23,66 a10 A1 & 26,93 a6 A1 & 19,29 a7 $\mathrm{A} 1$ & 20,62 a 5 A1 & 4,24 a7 A1 & 5,01 a10 A1 \\
\hline IAC 82-3111 & 26,25 a12 A1 & 28,72 a7 A1 & 20,97 a 9 A1 & 24,06 a 9 A1 & 3,95 a 6 A 1 & 4,30 a5 A1 \\
\hline SP79-2312 & 21,90 a7 A1 & 24,93 a4 A1 & 17,50 a5 $\mathrm{A} 1$ & 19,61 a 4 A1 & 3,66 a5 A1 & 3,51 a2 A1 \\
\hline SP71-6180 & 23,62 a10 A1 & 26,12 a5 A1 & 18,27 a 6 A1 & 22,09 a7 A1 & 3,97 a 6 A1 & 4,64 a8 A1 \\
\hline SP79-2233 & 21,56 a 6 A1 & 27,34 a6 A2 & 18,92 a 6 A1 & 22,97 a 8 A 1 & 3,62 a5 A1 & 4,52 a7 A1 \\
\hline RB78-5750 & 22,67 a 8 A 1 & 26,79 a6 A2 & 19,64 a7 $\mathrm{A} 1$ & 22,22 a7 A1 & 3,76 a 6 A 1 & 4,79 a9 A2 \\
\hline SP72-1861 & 23,38 a 9 A1 & 25,15 a4 A1 & 21,34 a 9 A1 & 22,33 a7 A1 & 3,79 a 6 A 1 & 4,80 a9 A2 \\
\hline RB80-6043 & 24,09 a10 A1 & 23,24 a1 A1 & 20,39 a 8 A1 & 21,11 a 6 A1 & 3,11 a3 A1 & 3,80 a3 A1 \\
\hline CB $45-3$ & 25,14 a11 A1 & 24,40 a3 A1 & 17,14 a5 $\mathrm{A} 1$ & 21,43 a 6 A1 & 3,67 a5 A1 & 3,83 a3 A1 \\
\hline IAC 81-3049 & 23,87 a10 A1 & 27,46 a6 A1 & 19,01 a 6 A1 & 23,80 a 9 A2 & 3,58 a 4 A 1 & 4,89 a9 A2 \\
\hline IAC $80-3010$ & 25,42 a11 A1 & 30,58 a9 A2 & 19,46 a7 A1 & 22,38 a7 A1 & 3,73 a5 A1 & 5,72 a13 A2 \\
\hline IAC $83-4157$ & 21,77 a 6 A1 & 26,19 a5 A2 & 19,94 a 8 A1 & 18,15 a3 A1 & 3,05 a2 A1 & 3,73 a3 A1 \\
\hline IAC $86-1054$ & 24,15 a10 A1 & 25,40 a 4 A1 & 18,96 a 6 A1 & 22,12 a7 A1 & 4,63 a9 A1 & 6,37 a14 A2 \\
\hline IAC $83-4531$ & 24,85 a11 A1 & 24,80 a3 A1 & 21,23 a 91 & 23,29 a 8 A1 & 3,17 a3 A1 & 4,97 a10 A2 \\
\hline IAC51-205 & 25,04 a11 A1 & 25,23 a4 A1 & 21,02 a9 A1 & 18,93 a3 A1 & 3,55 a 4 A 1 & 4,70 a 8 A2 \\
\hline SP70-1078 & 27,15 a13 A1 & 28,09 a7 A1 & 23,87 a10 A1 & 24,16 a 9 A1 & 4,20 a7 A1 & 4,77 a9 A1 \\
\hline IAC 85-3229 & 23,19 a9 A1 & 25,53 a4 A1 & 17,55 a5 A1 & 21,74 a6 A1 & 3,50 a 4 A 1 & 4,44 a6 A1 \\
\hline IAC 87-3187 & 25,48 a11 A1 & 25,53 a4 A1 & 21,06 a9 A1 & 20,38 a5 A1 & 4,11 a7 A1 & 4,61 a7 A1 \\
\hline IAC $87-1365$ & 23,02 a 8 A1 & 24,17 a2 A1 & 19,23 a7 A1 & 20,71 a5 $\mathrm{A} 1$ & 3,55 a 4 A 1 & 3,91 a3 A1 \\
\hline
\end{tabular}

Continua 
Tabela 4. Conclusão

\begin{tabular}{|c|c|c|c|c|c|c|}
\hline \multirow{2}{*}{ Cultivar } & \multicolumn{2}{|c|}{$\%$ CEL } & \multicolumn{2}{|c|}{$\%$ HEM } & \multicolumn{2}{|c|}{$\%$ LIG } \\
\hline & 12 meses & 18 meses & 12 meses & 18 meses & 12 meses & 18 meses \\
\hline SP70-1143 & 23,96 a10 A1 & 26,81 a6 A1 & 19,85 a7 A1 & 20,50 a5 A1 & 4,36 a 8 A1 & 3,80 a3 A1 \\
\hline RB83-5486 & 23,72 a10 A1 & 24,62 a3 A1 & 17,72 a5 $\mathrm{A} 1$ & 21,41 a 6 A1 & 3,20 a3 A1 & 3,94 a3 A1 \\
\hline IAC 83-4107 & 22,71 a 8 A1 & 23,69 a2 A1 & 18,88 a 6 A 1 & 20,16 a5 A1 & 3,64 a5 A1 & 4,10 a 4 A1 \\
\hline IAC 86-2210 & 22,89 a8 A1 & 23,17 a1 A1 & 21,24 a9 A1 & 20,42 a5 $\mathrm{A} 1$ & 3,90 a 6 A1 & 4,79 a9 A1 \\
\hline IAC 83-1144 & 27,98 a14 A1 & 26,39 a5 A1 & 19,69 a7 A1 & 22,06 a7 A1 & 4,23 a7 $\mathrm{A} 1$ & 4,21 a5 A1 \\
\hline IAC 82-2045 & 22,43 a8 A1 & 30,58 a9 A2 & 19,08 a 6 A1 & 28,86 a10 A2 & 3,45 a 4 A 1 & 5,40 a12 A2 \\
\hline IAC $87-3184$ & 24,70 a11 A1 & 26,28 a5 A1 & 17,41 a5 A1 & 20,92 a5 A1 & 3,88 a 6 A 1 & 4,57 a7 A1 \\
\hline IAC 82-3092 & 28,56 a15 A1 & 25,24 a 4 A1 & 14,53 a3 A1 & 21,23 a 6 A2 & 5,60 a $11 \mathrm{~A} 1$ & 5,20 a11 A1 \\
\hline IAC 86-1061 & 23,41 a9 A1 & 25,77 a5 A1 & 19,66 a7 A1 & 22,00 a7 $\mathrm{A} 1$ & 3,92 a6 A1 & 4,59 a7 A1 \\
\hline IAC 83-2045 & 25,70 a12 A1 & 23,63 a2 A1 & 20,17 a 8 A1 & 20,25 a5 A1 & 4,41 a 8 A 1 & 4,72 a 8 A1 \\
\hline IAC 86-1056 & 29,01 a15 A2 & 24,99 a4 A1 & 13,41 a2 A1 & 21,63 a6 A2 & 4,53 a 9 A1 & 4,97 a10 A1 \\
\hline IAC $83-4128$ & 24,97 a11 A1 & 28,35 a7 A1 & 19,12 a 6 A1 & 23,93 a9 A2 & 4,19 a7 A1 & 5,32 a12 A2 \\
\hline IAC 87-1017 & 22,77 a8 A1 & 22,93 a1 A1 & 19,60 a7 A1 & 21,39 a6 $\mathrm{A} 1$ & 7,11 a12 A2 & 4,93 a10 A1 \\
\hline IAC 81-1019 & 25,85 a12 A1 & 26,75 a 6 A1 & 23,05 a10 A1 & 20,74 a5 A1 & 4,18 a7 A1 & 4,90 a9 A1 \\
\hline SP71-1406 & 21,43 a6 A1 & 26,33 a5 A2 & 25,02 a11 A1 & 21,19 a 6 A1 & 2,94 a 2 A1 & 3,85 a3 A1 \\
\hline IAC 87-1392 & 26,74 a13 A1 & 25,98 a5 A1 & 23,41 a10 A1 & 23,03 a 8 A 1 & 4,19 a7 A1 & 4,01 a4 A1 \\
\hline IAC 83-2285 & 26,52 a13 A1 & 29,75 a 8 A1 & 23,61 a10 A1 & 21,25 a 6 A1 & 5,37 a10 A1 & 5,39 a12 A1 \\
\hline Média & \multicolumn{2}{|c|}{24,48} & \multicolumn{2}{|c|}{19,90} & \multicolumn{2}{|c|}{4,16} \\
\hline CV $(\%)$ genótipo & \multicolumn{2}{|c|}{9,53} & \multicolumn{2}{|c|}{11,96} & \multicolumn{2}{|c|}{12,97} \\
\hline $\mathrm{CV}(\%)$ idade & \multicolumn{2}{|c|}{10,05} & \multicolumn{2}{|c|}{13,68} & \multicolumn{2}{|c|}{14,93} \\
\hline
\end{tabular}

Médias seguidas de letras minúsculas e números distintos, na coluna, diferem pelo teste de Scott-Knott $(1974)$, com (P < 5\%). Médias seguidas de letras maiúsculas e números distintos, na linha, diferem pelo Teste de Tukey, com $(P<0,05)$.

Para os teores de CEL, nos 15 genótipos (SP711406, SP71-6192, SP79-2233, IAC80-3010, IAC81-1050, IAC81-2004, IAC82-1004, IAC82-2045, IAC83-1313, IAC83-2396, IAC83-4157, IAC86-1034, IAC87-3420, RB785750 e RB855036) observaram-se teores maiores na idade de 18 meses, enquanto os demais apresentaram teores de CEL semelhantes nas idades de 12 e 18 meses.

A resposta dos genótipos que apresentaram maiores teores de CEL na idade de 18 meses foi contrária à de BANDA e VALDEZ (1976), KUng Jr. e Stanley (1982), Carvalho (1992) e Oliveira (1996) que determinaram a composição de cana-de-açúcar de 6 aos 24 meses, verificando redução no teor de CEL com o avanço na idade da cana-de-açúcar.

Como observado para os teores de FDN e FDA, também para o teor de CEL, de maneira geral, foram maiores para a idade de 18 meses. Quanto aos genótipos, nas idades de 12 e 18 meses, eles se discriminaram em 15 e 9 grupos respectivamente. Pode-se verificar, ainda, forte tendência de que os grupos com maiores teores de FDN e FDA mostraram também maiores teores de CEL, sugerindo que nesses a digestibilidade da matéria seca seria menor.

Em 14 dos 60 genótipos estudados (SP71-6192, IAC52-150, IAC66-6, IAC81-3049, IAC82-1004, IAC822045, IAC82-3092, IAC83-1313, IAC83-2396, IAC83-4128, IAC85-1004, IAC86-1056, IAC87-3420 e RB855036) os teores de HEM foram maiores na idade de 18 meses; desses, sete mostraram aumento do teor de CEL na idade de 18 meses em comparação aos 12; os outros 46 genótipos apresentaram teores de HEM semelhantes nas duas idades de corte. BANDA e VALDEZ (1976), Kung Jr. e Stanley (1982), Carvalho (1992) e Oliveira (1996) determinaram a composição de canade-açúcar de 6 aos 24 meses, verificando redução para esse teor com a maturidade.

A exemplo do que ocorreu para os outros componentes da fibra, também o teor de HEM da idade de 18 meses foi, no geral, maior que aquele da idade de 12 meses. Ainda quanto ao teor de HEM os 60 genótipos foram separados, respectivamente, em 11 e 10 grupos diferentes nas idades de 12 e 18 meses. Diferentemente de FDN, FDA, e CEL, os genótipos dos grupos com maiores teores de HEM aparecem mais distribuídos no perfil da Tabela 4, mostrando que genótipos com maior teor de HEM poderiam ter maior digestibilidade da matéria seca.

Quanto ao teor de LIG, observaram em 21 genótipos (SP71-6192, SP72-1861, SP80-1842, IAC51205, IAC52-150, IAC80-3010, IAC81-1050, IAC81-2004, IAC81-3049, IAC82-1004, IAC82-2045, IAC82-3258, IAC83-4128, IAC83-4531, IAC84-1042, IAC86-1034, IAC86-1054, IAC87-3396, IAC87-3420, RB785750 e RB855036) teores de LIG maiores na idade de 18 meses. Dentre eles, os genótipos SP72-1861, SP80-1842, IAC51-205, IAC83-4531, IAC84-1042, IAC86-1054, IAC87-3396 mostraram aumento somente no teor de LIG, enquanto nos demais houve aumento nos teores de CEL e/ou HEM. Os demais genótipos apresentaram teores de LIG semelhantes nas idades de 12 e 18 meses. 
O aumento do teor de LIG aos 18 meses foi contrário ao resultado obtido por BANDA e VALDEZ (1976), Kung Jr. e Stanley (1982), Carvalho (1992) e Oliveira (1996) que determinaram a composição de cana-de-açúcar de 6 aos 24 meses, observando redução desse teor com a maturidade.

Também para o teor de LIG, de modo geral, os teores dos genótipos cortados na idade de 18 meses foi maior que aqueles de 12 meses. Assim, pode-se dizer que todos os componentes da fibra, de maneira geral, apresentaram teores mais elevados na idade de 18 meses.

\section{CONCLUSÕES}

1. Os teores de matéria seca e proteína bruta dos 60 genótipos foram maiores na idade de 12 meses, enquanto o teor de matéria mineral foi maior aos 18 meses; os teores de extrato etéreo foram semelhantes nas duas idades.

2. Para os teores de carboidratos totais não estruturais, fibra insolúvel em detergente neutro, fibra insolúvel em detergente ácido, celulose, hemicelulose e lignina houve diferença entre as idades de 12 e 18 meses, com tendência a ser maior na segunda idade na maioria dos genótipos, exceto para os teores de carboidratos totais não extruturais.

3. Com vistas à alimentação animal, sugerese separar os grupos de genótipos que mostraram maiores teores de carboidratos totais não estruturais e baixos teores dos componentes da fibra, nas idades de 12 e 18 meses.

\section{REFERÊNCIAS}

BANDA, M.; VALDEZ, R.E. Effect of stage of maturity on nutritive value of sugar cane. Tropical Animal Production, Santo Domingo, v.1, p.94-97, 1976.

BOIN, C.; MATTOS, W.R.S.; D’ARCE, R.D. Cana-de-açúcar na alimentação de ruminantes. In: PARANHOS, S.B. Cana-deaçúcar: cultivo e utilização. Campinas: Fundação Cargill, 1987. v.II, p.805-856.

CARVALHO, G.J. Avaliação do potencial forrageiro e industrial de variedades de cana-de-açúcar (ciclo de ano) em diferentes épocas de corte. 1992. 63f. Dissertação (Mestrado)ESAL, Lavras.

GOERING, H.K.; SOEST, P.J. van. Forage Fiber Analyses: Apparatus, reagents, procedures, and some applications. Washington: USDA, 1970. 20p. (Agriculture Handbook, 379).
KUNG Jr., K.; STANLEY, R.W. Effect of stage of maturity on the nutritive value of wholeplant sugarcane preserved as silage. Journal Animal Science, Albany, v.54, p.689-695, 1982.

LOVADINI, L.A.; MORAES, C.L.; PARANHOS, S.B. Levantamento sobre a composição química bromatológica de 39 variedades forrageiras de cana-de-açúcar. Anais da E.S.A. “Luiz de Queiroz", Piracicaba, v.24, p.189-198, 1967.

MATSUOKA, S.; HOFFMANN, H.P. Variedade de cana de açúcar para bovinos. In: SIMPÓSIO SOBRE NUTRIÇÃO DE BOVINOS, 5., 1993, Piracicaba. Anais... Piracicaba: FEALQ, 1993. p.17-35.

MOREIRA, H.A. Cana-de-açúcar na alimentação de bovinos. Informe Agropecuário, Belo Horizonte, v.9, p.14-16, 1983.

NUNES JUNIOR., D.M.S. Variedades de cana-de-açúcar. In: PARANHOS, S.B. Cana-de-açúcar: cultivo e utilização. Campinas: Fundação Cargill, 1987. v.II, p.187-259.

OLIVEIRA, M.D.S.; SAMPAIO, A.A.M.; CASAGRANDE, A.A., et al. Estudo da composição químico-bromatológica de algumas variedades de cana-de-açúcar. In: REUNIÃO ANUAL DA SOCIEDADE BRASILEIRA DE ZOOTECNIA,33., 1996, Fortaleza. Anais... Fortaleza: Sociedade Brasileira de Zootecnia, 1996. v.2, p.314.

SALLAS, M.; AUMONT, G.; BIESSY, G.; MAGNIE, E. Effect of variety, stage of maturity and nitrate fertilization on nutritive values of sugar canes. Animal Feed Science and Technology, v.39, p.265-277, 1992.

SCOTT, A.J., KNOTT, M. A cluster analysis method for grouping means in the analyses of variance. Biometrics, Washington, v.30, p.507-512, 1974.

SILVA, D. J. Análises de Alimentos: métodos químicos e biológicos. Viçosa: Editora Universidade Federal de Viçosa, 1998. 165p. 